\title{
Formación del profesorado en educación física e inclusión del alumnado con pluridiscapacidad
}

\author{
Teresa LLEIXÀ ARRIBAS \\ Merche RÍOS HERNÁNDEZ
}

Datos de contacto:

Teresa Lleixà Arribas

Universidad de Barcelona

teresa.Ileixa@ub.edu

Merche Ríos Hernández

Universidad de Barcelona

Mercherios@ub.edu

\section{RESUMEN}

La presente investigación pretende analizar los procesos formativos de un grupo de maestros y maestras en activo que, además de su actividad docente en los centros escolares, participan como dinamizadores y observadores en un programa de actividad físico-deportiva fuera del horario lectivo que comporta la inclusión de alumnado con pluridiscapacidad. Los objetivos se concretan en: Identificar y analizar situaciones de práctica que generan reflexión en el profesorado; y valorar, en las reflexiones de los maestros y maestras participantes, cómo la experiencia en el programa promueve su desarrollo profesional. Se ha seguido una metodología cualitativa de estudio de caso, utilizando como técnicas de investigación la observación participante y el grupo compartido de reflexión. Se han analizado 49 diarios de sesiones, correspondientes a dos cursos académicos. Los resultados muestran que el profesorado de la experiencia ha podido reforzar sus actitudes inclusivas al debatir sobre las barreras y facilitadores de la participación sin límites. Se reconoce la relevancia del clima de aula y las emociones como elementos clave para la inclusión. Numerosas estrategias docentes para dar respuesta a las diferentes situaciones tienen que ver con orientaciones didácticas, tipología de las actividades, adaptaciones y criterios de seguridad. El apoyo de las familias resulta valioso, rompiendo barreras y creando conciencia social. Con todo ello, la necesidad de repensar el modelo de las actividades físicas extraescolares es una de las principales conclusiones del estudio.

PALABRAS CLAVE: Educación física; actividades extraescolares; inclusión; discapacidad; pluridiscapacidad. 


\title{
Teacher training in physical education and inclusion of students with multiple disabilities
}

\begin{abstract}
This research aims to analyse the training processes of a group of in-service teachers who participated as teachers and observers in an extracurricular program of sport and physical activity. Students with and without multiple disabilities took part in this program. The objectives were: to Identify and analyse situations that generate teacher reflection; and to assess how the experience in this program promotes professional development. A qualitative case study methodology has been followed. Participant observation and critical reflection group were used as research techniques. 49 field journals have been analysed, corresponding to two academic courses. The results show that the teachers have been able to strengthen their inclusive attitudes by discussing barriers and facilitators of participation without limits. In addition, the classroom climate and emotions are presented as key elements for inclusion. Teaching strategies for inclusion are related to teaching guidelines, type of activities, adaptations and safety criteria. Family support is important, breaking down barriers and creating social awareness. Taking into account the results, the need to rethink the model of extracurricular physical activities arises as the main conclusion of the study.
\end{abstract}

KEYWORDS: Physical education; extracurricular physical activities; inclusion; disability; multiple disabilities

\section{Introducción}

La educación inclusiva, entendida como un proceso destinado a responder a la diversidad del alumnado aumentando su participación y reduciendo la exclusión escolar y social (UNESCO, 2005), se ha extendido como posición ideológica y pedagógica por su carácter ético y de respuesta al derecho universal a la educación. La inclusión implica cambio, así como reestructuración de concepciones políticas y escolares para garantizar una formación de calidad para todo el alumnado. Va más allá de incrementar la participación, puesto que busca logros reales de aprendizaje (Booth \& Ainscow, 2002; Naciones Unidas, 2006; Unesco, 2009) y requiere una formación docente acorde con estas premisas.

La presente investigación pretende analizar los procesos formativos de un grupo de maestros y maestras en activo que además de su actividad docente en los centros escolares, participan como dinamizadores y observadores en un programa de actividad física fuera del horario lectivo que comporta la inclusión de alumnado con pluridiscapacidad

La inclusión del alumnado con discapacidad en educación física 
comparte los mismos presupuestos ideológicos y pedagógicos de la educación inclusiva. Se entiende que la educación fisica dirigida a favorecer un desarrollo desde sus diferentes dimensiones física, cognitiva, emocional y afectiva y beneficiosa para la mejora de la salud a lo largo de la vida, debe atender a todo el alumnado por igual, evitando la injusticia que representaría privar de dichos beneficios a quienes presentan características o capacidades diferentes.

$\mathrm{Su}$ implementación en la escuela preocupa no solo a docentes, sino también a investigadores, como muestran los numerosos artículos sobre revisiones sistemáticas relativas a esta temática, entre las que podemos señalar: Block \& Obrusnikova (2007); Rekaa, Hanisch, \& Ytterhus, (2018); Hernández-Álvarez, Fernández-Cabrera, Álvarez-Pérez, y LópezAguilar (2019); Pocock \& Miyahara (2018); Qi, J., \& Haa, A.S. (2012).

La mayoría de los estudios sobre inclusión en educación física, según Block \& Obrusnikova (2007), versan sobre: el soporte que recibe el alumnado con discapacidad; la sensibilización de los compañeros; cómo afecta la inclusión en el entorno educativo; las actitudes del alumnado sin discapacidad; las interacciones sociales; el tiempo académico de aprendizaje; la formación y las actitudes del profesorado.

Atendiendo a la tipología de los estudios sobre inclusión en educación física observamos, coincidiendo con Rekaa, Hanisch, \& Ytterhus, (2018), que la mayoría puede agruparse según hagan referencia a las actitudes del profesorado o a las experiencias inclusivas. En relación con estas últimas se hará referencia a las primeras, más adelante, en el apartado de formación del profesorado-, cabe señalar que muestran la necesidad de reorientar la práctica pedagógica respetando las individualidades y potencialidades de todo el alumnado. En este sentido, Claverol (2000) destaca la importancia del diagnóstico previo y la información del contexto para el profesorado y De Souza \& Pich, (2013) indican la necesidad de un replanteamiento del concepto de desarrollo motor normal y de la evaluación.

El clima del aula en estas experiencias condiciona necesariamente el éxito de las mismas, destacando para ello la importancia de la sensibilización de compañeros y agentes educativos (Felipe-Rello, Garoz, Tejero-González, 2020; Garro, Median, Fernández-Río, 2002; Reina, Hutzler, Iniguez-Santiago, Moreno-Murcia, 2016). Dicha sensibilización suele realizarse mediante la práctica de actividades físicas adaptadas en las que se prioriza la vivencia y la experiencia en situaciones diferentes.

Los estudios sobre estrategias facilitadoras de la inclusión (Fernández, Sánchez, Jiménez, Navaro, Anguera, 2012; Ríos, 2006, 2009) destacan las adaptaciones de las tareas, el aprendizaje cooperativo y la enseñanza multinivel como medidas para permitir la participación activa y efectiva de todo el alumnado. En relación con las tareas multinivel Petrie, Devcich, \& Fitzgerald, (2018) indican que requieren conocer bien al alumnado, y 
reflexionar constantemente sobre la práctica, entendiendo las posibilidades de aprendizaje más que las limitaciones.

También hay que velar para que la educación física no vaya aislada sino que haya una coordinación de todo el centro educativo (Mendoza, 2009) y acciones colaborativas entre el profesorado, así como apoyo de las familias (Pocock \& Miyahara, 2018).

En la revisión de la literatura, sin embargo, no resulta fácil encontrar estudios de inclusión de alumnado con pluridiscapacidad en educación física o deportiva, más allà de artículos de difusión de la experiencia que contextualiza la presente investigación (Ríos et al., 2019). Los estudios sobre esta temática tienen más que ver con la utilización de programas psicomotores en la rehabilitación de estos niños y niñas como, por ejemplo, Danelciuc, Betiuc \& Danelciuc (2012). La pluridiscapacidad y el derecho a la inclusión en la educación física y el ocio deportivo se debatió, sin embargo, en el 1er Encuentro pedagógico sobre buenas prácticas en la inclusión deportiva de la Universidad de Barcelona (Ríos y Panchón, eds., 2018). Se puso aquí de manifiesto la necesidad de analizar los aspectos críticos que garanticen la presencia, la participación y el aprendizaje de todo el alumnado sin limites, mediante la reflexión sobre dos experiencias de inclusión de alumnado con pluridiscapcidad, una de las cuales es el programa Juguem! [jJuguemos!] que contextualiza el presente artículo.

El programa Juguem! (Rios et al., 2019), fue impulsado en el curso 20152016, por el Institut Barcelona Esports (IBE) del Ayuntamiento de Barcelona, en el marco del programa L'Esport Inclou [E1 Deporte incluye], y realizado con la colaboración de diversas entidades locales: Escuela Els Llorers; Asociación de Familias del Alumnado de la misma; Asociación Deportiva Eixample; Asociación y Fundación de Parálisis Cerebral ASPACE; el grupo de estudio Educació Física $i$ la inclusió de l'alumnat amb discapacitat del Instituto de Ciencias de la Educación de la Universidad de Barcelona; y la Asociación deportiva JOCVIU.

Desde su segunda edición forma parte de la oferta habitual de actividades fisico-deportivas que propone la Asociación Deportiva Eixample fuera del horario escolar. Se muestra como un proyecto consolidado, tras su cuarto año de aplicación, habiéndose replicado en otras entidades deportivas de la ciudad de Barcelona.

Constituye un programa de actividad físico-deportiva fuera del horario lectivo que, bajo los principios del Diseño Universal para el Aprendizaje (McGuire, Scott \& Shaw, 2006) y mediante la metodología cooperativa y la estimulación sensorial, posibilita la inclusión de dos alumnos con pluridiscapacidad, junto con compañeros y compañeras sin discapacidad, en igualdad de condiciones y sin segregación alguna, consiguiendo dar respuesta a las diferentes necesidades educativas de todo el grupo en un espacio de total convivencia. 
El grupo de participantes está formado por 17 alumnos y alumnas de quinto y sexto de primaria, entre ellos dos alumnos con pluridiscapacidad que presentan ambos parálisis cerebral además de discapacidad intelectual severa, y precisan de silla de ruedas para el desplazamiento. Son totalmente dependientes en las actividades de la vida diaria: vestirse, asearse, alimentarse, desplazarse, entre otros aspectos. Uno de ellos presenta movimientos distónicos (movimientos involuntarios de sus extremidades) y tiene discapacidad visual añadida (S). El otro tiene hipotonía muscular, aunque realiza movimientos voluntarios e incluso es capaz de realizar marcha acompañada. También presenta ciertos rasgos de trastorno del espectro autista $(\mathrm{P})$.

Al tratar la inclusión el alumnado con pluridiscapacidad surge un tema clave que es el de la capacitación del profesorado. La formación previa específica y el contacto con experiencias inclusivas son factores determinantes para que los docentes sean competentes a la hora de favorecer entornos de educación física inclusiva. La formación docente resulta crucial para incrementar los recursos del profesorado (Caus, y Santos, 2011), mejorar las percepciones relativas a su propia competencia (Diaz del Cueto, 2009; Reina, Hemmelmayr, y Sierra Marroquín, 2016)) y mejorar las actitudes (Elliott, 2008; Raimundo, 2014; Seçer, 2010).

Las actitudes del profesorado resultan una cuestión determinante en la inclusión del alumnado con discapacidad en educación física. Así, es fácil comprender que en el estudio de Haegele, Zhu, \& Davis (2018), sobre barreras y facilitadores de la participación, se presente la actitud del profesorado encabezando tanto la categoría de barreras, como la de facilitadores. Para que estas actitudes sean facilitadoras es necesario que se modifiquen creencias sobre las posibilidades inclusivas de la educación física, mediante actividades vivenciales y experienciales (Lleixà et. al., 2017). Las actividades experienciales en las que se reflexione colectivamente sobre la acción son también apuntadas por Valencia-Peris, Mínguez-Alfaro, y Martos-García (2020) como las más indicadas para esta formación, destacando estrategias como la investigación acción, las simulaciones o los talleres de acción directa.

Como ya se ha indicado, la presente investigación pretende analizar los procesos formativos de un grupo de maestros y maestras en activo, que además de su actividad docente en los centros escolares, participan como dinamizadores y observadores en las sesiones de actividad fisica en el "Proyecto Juguem" de inclusión de alumnado con pluridiscapacidad. Los objetivos del estudio se concretan en: a) Identificar y analizar situaciones de práctica que generan reflexión en el profesorado; b) Valorar, en las reflexiones de los maestros y maestras participantes, cómo la experiencia "Juguem" promueve su desarrollo profesional. 


\section{Método}

El presente estudio se enmarca en una metodología cualitativa de estudio de caso, utilizando como técnicas de investigación la observación participante y el grupo compartido de reflexión. El estudio de caso se contextualiza en la experiencia "Juguem" descrita en la introducción del artículo.

\section{Participantes}

Los participantes en la investigación han sido, por un lado, el alumnado participante de la experiencia: 17 alumnos y alumnas de cuarto y quinto de primaria, dos de los cuales son alumnos con pluridiscapacidad. Por otro lado, 7 maestras y 3 maestros pertenecientes al Grupo de estudio en Educación Física e inclusión del Instituto de Desarrollo Profesional-ICE de la Universidad de Barcelona, así como la profesora (Investigadora) coordinadora de dicho grupo.

Tabla 1

Características de los maestros y maestras participantes en la investigación

\begin{tabular}{cccc}
\hline Maestro/a & Género & $\begin{array}{c}\text { Años de } \\
\text { experiencia } \\
\text { docente }\end{array}$ & $\begin{array}{c}\text { Años de } \\
\text { experiencia en EF } \\
\text { e Inclusión }\end{array}$ \\
\hline 1. & $\mathrm{M}$ & 34 & 34 \\
\hline 2. & $\mathrm{M}$ & 23 & 23 \\
\hline 3. & $\mathrm{M}$ & 10 & 5 \\
\hline 4. & $\mathrm{H}$ & 8 & 4 \\
\hline 5. & $\mathrm{H}$ & 6 & 3 \\
\hline 6. & $\mathrm{H}$ & 11 & 13 \\
\hline 7. & $\mathrm{M}$ & 15 & 17 \\
\hline 8. & $\mathrm{H}$ & 1 & 8 \\
\hline 9. & $\mathrm{H}$ & 17 & \\
\hline 10. & $\mathrm{M}$ & 8 & 17 \\
\hline
\end{tabular}

\section{Técnicas de recogida de la información y análisis}

La información se ha recogido mediante el diario de las sesiones realizado de forma narrativa y estructurado en dos partes. La primera parte corresponde a las observaciones realizadas durante la sesión y recoge fundamentalmente incidentes críticos (Bilbao y Monereo, 2011; Flanagan, 1954). La segunda parte corresponde a las reflexiones e impresiones del grupo de maestros y maestras en el grupo compartido de reflexión de después de la sesión. 
E1 análisis se ha realizado de forma inductiva utilizando como instrumento de apoyo el software de análisis de datos cualitativos N-Vivo.

\section{Procedimiento}

Las sesiones se imparten durante el curso escolar, los miércoles de 18 a 19.30h, en la Escuela Els Llorers de Barcelona Sistemáticamente, durante el desarrollo de las sesiones, dos o tres personas del grupo de maestros ejercen de dinamizador o dinamizadora, mientras que el resto realiza la observación. Las sesiones tienen una duración de 90 minutos durante los cuales se realizan actividades fisico-deportivas y finalizan con una asamblea en la que participan todos los niños y niñas. Al finalizar la sesión, tras despedir a los niños y niñas, el grupo de maestros y maestras permanece in situ realizando un debate reflexivo que puede extenderse hasta una duración de 60 minutos.

El diario es redactado por una de las personas observadoras y recoge los incidentes críticos de la sesión y las principales ideas desarrolladas en el debate reflexivo.

Se han analizado 49 diarios, con una extensión de dos a cuatro folios para cada sesión, 24 diarios redactados durante el curso 2015-16 y 25 durante el curso 2016-17. En el análisis se han seleccionado los párrafos codificándolos en los nodos correspondientes, determinados por las categorias provenientes del análisis inductivo. Se ha partido de cinco dimensiones establecidas a partir de los objetivos del estudio y de la revisión de la literatura. El análisis inductivo ha dado lugar a nuevas categorías emergentes (Tabla 2). Los resultados se presentan en función de dichas dimensiones.

Tabla 2

Categorias provenientes del análisis inductivo

\begin{tabular}{|l|l|}
\hline Dimensiones & \multicolumn{1}{|c|}{ Categorias } \\
\hline \multirow{4}{*}{ Actitudes inclusivas } & Actitudes de participantes \\
\cline { 2 - 2 } & Participación sin limites \\
\cline { 2 - 2 } Clima de aula & Transferencia social \\
\hline \multirow{4}{*}{ Estrategias docentes } & Ambiente \\
\cline { 2 - 2 } & Emociones \\
\cline { 2 - 2 } & Interacciones \\
\cline { 2 - 2 } & Orientaciones didácticas \\
\cline { 2 - 2 } & Actividades \\
\cline { 2 - 2 } & Adaptaciones \\
\hline Familias & Reguridad \\
\hline Desarrollo profesional & Caracterínticas docentes deseables \\
\hline & \\
\hline
\end{tabular}




\section{Dilemas e incógnitas}

Conciencia de aprendizaje

\section{Consideraciones éticas}

Las familias del alumnado, así como el profesorado participante, han dado su consentimiento informado para la difusión de los resultados de la investigación resultante de la experiencia.

\section{Resultados}

Se presentan, a continuación, los resultados del análisis según las dimensiones del estudio. Las referencias que se muestran como evidencias llevan la codificación siguiente: $\left[\mathrm{N}^{\circ}\right.$ de sesión/Año de la experiencia- $\mathrm{N}^{\circ}$ de la referencia].

\section{Actitudes inclusivas}

En el presente apartado se analizan las evidencias que muestran de qué manera los maestros y maestras mejoran sus actitudes inclusivas mediante la reflexión sobre las diversas situaciones.

Los maestros y maestras participantes valoran reiteradamente la plena participación de todos los niños y niñas que forman parte de la actividad, así como la autonomía de los mismos en la realización de actividades y en la interacción entre los participantes con y sin pluridiscapacidad.

... la imagen es espectacular: todos los niños y niñas corriendo como locos por la pista, riendo y disfrutando. [S02/2-R1]

Durante toda la sesión se ha observado una gran autonomía por parte de los participantes, aunque tuvieran un adulto, que les ofrecia soporte, ellos participaban en todo momento de las distintas tareas, así mismo se ha visto incrementada la interacción con $\mathrm{P}$ y $\mathrm{S}$, principalmente con el aumento de contacto físico. [S17/1-R1]

De la misma manera, otorgan una especial atención al hecho de que sean los mismos niños y niñas los que lleguen a ser capaces de autogestionar las medidas para que la inclusión sea efectiva.

Es la primera sesión donde los participantes autogestionan los acompañamientos de S y P. Sin que nadie se lo mandara han cogido a $\mathrm{P}$ y S para iniciar el juego. [S08/1-R1]

...valoramos muy positivamente que uno de los éxitos de Juguem! es el de empoderar a los alumnos para que salgan de ellos mismos las adaptaciones inclusivas para la participación de todos sin distinción. [S08/2-R1]

El profesorado es, así mismo sensible a la evolución de las actitudes 
inclusivas del alumnado a lo largo de las diferentes sesiones.

Los niños/as empiezan a tener conciencia de las dificultades que pueden presentar S y P, puesto que empiezan a preguntar, a ver y a entender que van más lentos y que hay cosas que no pueden realizar del mismo modo. El primer día observaron y compartían espacio y juego, el segundo día empezaron a relacionarse, y el tercer día empiezan a pensar en ellos.[S03/1$\mathrm{R} 1]$

Más adelante, el hecho que la discapacidad no se presente como un tema tabú también es valorado positivamente.

Nos parece muy destacable también que se hable abiertamente de la discapacidad, y que una niña comente que tiene un primo con parálisis cerebral. [S02/2-R2]

Se da también una gran naturalidad en aceptar las diferencias de $\mathrm{S}$ y $\mathrm{P}$ [S04/2-R2]

Un tema al que se llega inevitablemente es el de la participación sin límites. La participación sin límites se presenta como una respuesta a una necesidad social.

...un tipo de actividad como la que se genera en el proyecto da respuesta a una necesidad social, la posibilidad de que todo tipo de niños y niñas puedan participar en las actividades extraescolares deportivas. [S03/1-R1]

Se reconoce, sin embargo, la necesidad de recursos, más allá de los que actualmente disponen las escuelas, y de personal de apoyo.

La transferencia social del programa, a parte de la difusión que pueda realizarse para promocionarlo, se produce en el seno del mismo desde una doble perspectiva. Por un lado está el análisis que realizan los mimos niños y niñas, ya que en la asamblea final de las sesiones se tratan diferentes cuestiones, como por ejemplo: ¿por qué hay personas con discapacidad? ¿qué pasaría si S y P fueran a las clases de educación física en la escuela? Por otro lado, a lo largo de los años que ha durado la experiencia se han propuesto actividades conjuntas con otros colectivos, como por ejemplo un equipo que entrena futbol en el mismo recinto. También se han realizado sesiones en el parque cercano a la instalación.

Laura les pregunta sobre el objetivo de ir a jugar al parque y si hay algo que les preocupe. Salen a la luz comentarios como que el objetivo es demostrar a la gente que ellos (S y P )también pueden jugar con todos. Afirman también que otro objetivo puede ser enseñar a los demás cómo jugar con ellos (me encanta este comentario ya que se sienten expertos en jugar con $\mathrm{S}$ y $\mathrm{P}$ ). [S30/2-R1]

...para acabar juegan a la trituradora y nada más empezar algunos niños que están jugando por allí, al parecer amigos de Jo, se interesan por la dinámica y se ponen a jugar con ellos. Cuando acaba el juego Jo les presenta S y P. Es brutal [S30/2-R2] 


\section{Clima de aula}

En este apartado se analizan las evidencias que muestran las reflexiones en torno al clima de aula y las estrategias que aprenden los docentes para facilitarlo.

Crear un buen ambiente resulta uno de los objetivos para el buen desarrollo de las sesiones. El inicio de las sesiones viene encabezado por un túnel formado por los propios niños y niñas para ir recibiendo a quienes van llegando.

...el túnel de inicio de la sesión todos lo realizan en un buen ambiente de amabilidad y ganas de participar. Casi todos los participantes saludan expresamente a $\mathrm{P}$ y a $\mathrm{S}$ cuando pasan por el túnel [S22/2-R1]

Los calificativos que más se repiten para designar el ambiente son "tranquilo", "participativo", "dinámico", " calmado" y en menos ocasiones, "calido" y "distendido". Este tipo de ambiente es el deseado para el buen funcionamiento de las sesiones, de tal manera que encontramos reflexiones del tipo:

...la importancia de un ambiente cálido y distendido, jugar no es sinónimo de ruido[ $\mathrm{S} 03 / 1-\mathrm{R} 1]$

En alguna ocasión, sin embargo, se pone de manifiesto cómo los conflictos pueden ir en contra de la diversión. Los conflictos suelen ser comentados en la asamblea de final de la sesión, utilizándose este espacio para la reflexión.

Por otro lado, la incidencia de las emociones en el clima de aula también es un aspecto a destacar. Se observa que con la evolución de las actividades hay una evolución en los estados emocionales. Con cada cambio del tipo de actividades hay una primera fase de grandes expectativas, con mucha excitación, que se va suavizando. La valoración es que esta regulación no es negativa, sino natural y que no deja de haber ilusión en las sesiones.

En la dinamización se busca intencionadamente despertar emociones positivas de júbilo, alegría y satisfacción. El túnel de bienvenida con aplausos, consignas conjuntas de ánimos y actividades concretas para identificar estados de ánimos van dirigidas a esa finalidad.

Gemma, que hace de observadora en diversas actividades extraescolares, comenta que Juguem! es con diferencia la actividad donde los niños y niñas ríen más. [S15/2-R1]

Finalmente, cabe indicar que las interacciones entre los participantes son el tema que más referencias presenta en esta dimensión de análisis. A lo largo de los diarios de las sesiones se observa claramente como las interacciones van enriqueciéndose conforme avanza el curso. Así vemos en la primera sesión frases como:

La interacción entre los niños no se produce hasta muy avanzada la sesión y 
no es natural ni espontánea, algún adulto les pide esa interacción [S01/1-R1]

Y en la sesión 19:

El equipo coincide en que ha sido una muy buena sesión, donde las interacciones han sido muy predominantes. [S19/1-R1]

Potenciar las interacciones es una tarea intencionada entre quienes dinamizan la sesión, de tal manera que las progresiones pasan por:

...primero juego libre con los más próximos (libertad y experimentación), segundo aproximación con más compañeros (indicaciones para interactuar y consignas de juego) y por último más aumento de las interacciones jugando en gran grupo (propuestas grupales) [S13/1-R1]

Las interacciones con $\mathrm{P}$ y S son, en todo caso, las que más se destacan, en el sentido de que en las primeras sesiones son precisos recordatorios para facilitar dicha interacción, mientras que poco a poco se van haciendo espontáneas.

\section{Estrategias docentes}

De los diarios de las sesiones se desprenden numerosas orientaciones didácticas sobre las que reflexionar y que han quedado agrupadas en los nodos correspondientes esta dimensión. En este sentido, se le da una especial relevancia a aquellos elementos que conforman la sesión, juntamente con las tareas de predominio motor.

...dar especial importancia a aquello que va más allá del juego propiamente entendido (como tal): asambleas, rituales de entrada y salida, acompañamiento emocional, etc. [S03/1-R5]

La relación de $\mathrm{P}$ y $\mathrm{S}$ con el resto de niños y niñas también es objeto de numerosas reflexiones. En la primera sesión, se establece como objetivo romper el hielo y ofrecer tranquilidad a las familias, constatar que los participantes no están al servicio de S y P y que no se les considera como un experimento.

Las orientaciones didácticas que destacan tienen que ver con el acompañamiento de S y P. En primer lugar, están aquellas dirigidas a que los participantes tomen la iniciativa en el acompañamiento.

De nuevo se valora muy positivamente la estrategia de forzar acciones donde los participantes tengan que contar que alguien tiene que mover a $\mathrm{P}$ y a $\mathrm{S}$ sin que los adultos lo digan de inicio. Este hecho les hace conscientes de que si nadie lo hace no podrán participar [S06/0-R1]

Aparecen otras orientaciones más específicas, como por ejemplo: hablar con $\mathrm{P}$ y $\mathrm{S}$ antes de moverlos; la importancia del contacto corporal y con los materiales, para favorecer la estimulación táctil y la relación social; 
favorecer los relevos espontáneos para conducir las sillas de ruedas, ya que conducirlas supone un esfuerzo físico y mental.

Otras orientaciones hacen referencia a las agrupaciones. En este sentido, se indica que las agrupaciones se realizan libremente al inicio, para hacer que vayan cambiando poco a poco.

eliminar el NO a la hora de hacer las agrupaciones (no podéis jugar con...) y sugerirlo de forma positiva (aprovechad para jugar con aquellos compañeros con los que soléis estar y jugar poco) [S12/2-R3]

Las alusiones al material y recursos también son numerosas. A parte, de los materiales para adaptar la práctica como los "donuts" (material toroidal con ruedecitas) y las sillas de ruedas, se recalca la importancia del "plafón" para facilitar la comunicación con los participantes, o elementos para facilitar el contacto con $\mathrm{P}$ y S. Así mismo, se comenta la tranquilidad que ofrece la figura de la persona que realiza la tarea de auxiliar.

En cuanto a las actividades hay una orientación clara de iniciar el programa con actividades de cohesión de grupo, juegos sencillos o bien colaborativos de poca oposición, para poco a poco incrementar la complejidad de los retos y realizar actividades de oposición. Dicha progresión permite finalmente llegar a realizar actividades de iniciación deportiva. Sin embargo, en Juguem! no se valora el rendimiento deportivo y la competitividad es prácticamente inexistente.

Para los juegos y las actividades deportivas se proponen habitualmente adaptaciones que tienen que ver con: la utilización de materiales alternativos; acompañamientos; dar poderes mágicos; compensaciones, es decir, en caso de participantes que vayan más lentos aumentar la dificultad de los oponentes con actividades complementarias. Se valora que los mismos niños y niñas sean capaces de pensar las adaptaciones

Se valora muy positivamente los espacios de reflexión a los que los alumnos son invitados y acompañados por el docente dándoles la posibilidad de pensar las adaptaciones y cómo hacer para jugar; de esta forma se está acrecentando la iniciativa de los participantes de forma natural. [S12/1-R1]

Otro tipo de actividades diferentes son las de expresión corporal y danza donde apenas se requieren adaptaciones.

La primera actividad consiste en realizar una sencilla coreografia con ritmos africanos. Se ve a todo el mundo muy entretenido y $S$ y $P$ quedan perfectamente integrados sin ninguna modificación (excepto la consigna de que quién va con ellos de pareja los gire) [S17/2-R1]

Finalmente, el último bloque de estrategias tiene que ver con la seguridad. Los participantes reciben indicaciones de seguridad, sobre todo las relacionadas con la conducción de las sillas de ruedas, ya que es necesario que sean conscientes de que un mal uso del material puede ser peligroso y perjudicial. 
Se considera algo importante a trabajar con los niños y niñas, encontrando el equilibrio entre seguridad y descontrol. [S02/2-R2]

\section{Familias}

El trato de educadores con familias también requiere un aprendizaje. En este apartado se recogen las evidencias que indican de qué manera se progresa en esta relación con las familias.

Se resalta la implicación de las familias ya que se considera que la inclusión empieza por éstas

...para romper barreras hay que crear conciencia social; nosotros queremos hacerlo con las familias y con los participantes [S01/21- R3]

A este efecto, trimestralmente se organiza una sesión en la que participan las familias. De estas sesiones con familiares se destaca la evolución de las interacciones. Sobre todo se destaca el hecho de que las mamás de $\mathrm{P}$ y $\mathrm{S}$ también han evolucionado en sus comportamientos, al inicio, muy pendientes de sus hijos, y poco a poco participando de las sesiones como el resto de los familiares.

Se comenta la evolución que han sufrido las mamás de $\mathrm{P}$ y $\mathrm{S}$, al inicio pendientes de sus hijos jugando solo con ellos; a medida que avanza la sesión se han dejado llevar, suponemos que al ver que no era necesario que siempre estuvieron ellas, ya que si no había una persona había otra dispuesta a jugar con ellos. [S16/1- R3]

En general, hay un acuerdo en reconocer que los familiares viven un dia especial en estas sesiones compartidas y que se divierten rompiendo esquemas de las actividades deportivas que habian practicado en su infancia.

\section{Desarrollo profesional}

En la dimensión de desarrollo profesional se analiza la manera en que los maestros y maestras son conscientes de su formación.

El desarrollo profesional de los maestros y maestras que participan de la experiencia queda reflejado, en los diarios de las sesiones, mediante tres categorias de referencias diferentes. Por un lado, están aquellas referencias que expresan detalles de cómo esta experiencia les hace priorizar diferentes aspectos para ejercer la docencia. Un segundo grupo muestra dilemas e incógnitas que se plantean. Finalmente, en diversas ocasiones muestran la conciencia que tienen de estar aprendiendo.

Por lo que se refiere a las características de la docencia que se destacan estarian las que tienen que ver con la forma de interaccionar con el alumnado.

Una buena orientación didáctica extraída de las sesiones es la importancia 
del tono de voz y de la calma para crear un ambiente más adecuado [S02/1R2]

En diversas ocasiones se resalta la importancia que tiene saber interpretar qué está pasando en la sesión y actuar para dar buenas respuestas. Se ve como una característica importante la toma de decisiones por parte del docente.

se valora muy positivamente la sesión reportando el hecho de que la situación ha dado la vuelta a la planificación inicial. Saber detectar la necesidad del grupo y poder ofrecer la respuesta adecuada es el sí de nuestro trabajo. [S11/1- R1]

Otras formas de proceder deseables que se resaltan son la importancia de conocer a los niños y niñas y el papel que juegan dentro del grupo; o también la importancia de marcar pocos objetivos y concretos para ir evolucionando. Para acabar, se atribuye gran parte del éxito de las sesiones a la labor del trabajo colegiado.

En otro orden de cosas, a lo largo de los diarios aparecen diferentes dilemas e incógnitas que se han ido planteando. El principal dilema se establece entre la dimensión motriz - en el sentido de rendimiento- y la dimensión social del programa.

...reflexiona sobre la diferencia del paradigma del rendimiento físico que tiene como finalidad la mejora de la dimensión motriz contra el paradigma de la Educación Física educativa en el que están marcado nuestro proyecto. [S21/1- R1]

No se trata de despreciar un tipo u otro de aprendizaje sino de cuáles se quieren priorizar.

Otras incógnitas que aparecen en forma de preguntas en los diarios tienen que ver con: lo que es significativo para el alumnado; el papel que deben tener los maestros y maestras y escuela en el ambiente inclusivo; cómo mejorar la comunicación; los diferentes comportamientos de niños y niñas; el miedo a no cumplir las expectativas.

Por último, cabe indicar la conciencia que tienen los participantes de su aprendizaje, tanto por las decisiones que tienen que tomar durante la planificación y la realización de las sesiones como por las reflexiones posteriores.

En el equipo no hay egos, cada uno aporta lo mejor de sí mismo sin miedo al juicio ajeno y considera todo ello un aprendizaje personal y profesional brutal. [S03/2- R2]

\section{Discusión y conclusiones}

El trabajo colaborativo de un grupo de maestros y maestras, participando como dinamizadores y observadores en un programa de 
actividad fisica fuera del horario lectivo que incluye a alumnado con y sin pluridiscapacidad ha constituido un escenario valioso para el desarrollo profesional de este grupo de docentes. El análisis de sus reflexiones transcritas en un diario de campo ha permitido identificar las situaciones clave que crean debate y contrastarlas con otros estudios sobre esta temática. Hernández-Álvarez, Fernández-Cabrera, Álvarez-Pérez, y LópezAguilar (2019) insisten en la necesidad de incentivar la investigación sobre inclusión en educación fisica en y desde la práctica, premisa que se ha tenido especialmente en cuenta en el presente estudio.

El profesorado de la experiencia ha podido reforzar sus propias actitudes inclusivas debatiendo sobre dichas actitudes en el grupo, sobre las barreras y facilitadores de participación sin límites y sobre la manera en que la experiencia realizada puede tener una repercusión social. De este debate se deriva que favorecer la participación sin límites debería estar en el punto de mira de los programas de actividades extraescolares, dando respuesta a un requisito de justicia social. Sin embargo, la falta de recursos y de personal de apoyo, en la mayoría de los centros escolares, constituyen un obstáculo. Teniendo en cuenta, además, estudios como los de Haegele et al. (2018), quienes destacan la importancia del "equipamiento adaptado" y del "personal de soporte" como elementos facilitadores de la inclusión, se concluye que éste es un tema que debería estar en la lista de prioridades de las administraciones e instituciones educativas.

El clima de aula constituye otro de los elementos relevantes en la inclusión en educación física, en el que las emociones son un punto clave. Favorecer situaciones de júbilo y alegria en que se fomenten la interacciones entre todo el alumnado facilita sin duda la inclusión. Que los niños y niñas sean autónomos y generen por sí mismos dichas interacciones constituye un objetivo a tener en cuenta, para el cual pueden resultar útiles las actividades de sensibilización, dado que éstas facilitan las interacciones entre alumnado diverso. Tanto es así que Felipe-Rello, Garoz, y TejeroGonzález (2020) diseñan y validan un programa de sensibilización en educación física que se presenta como exitoso para este fin.

Dar respuesta a las diferentes situaciones que tienen lugar en la sesión conduce a crear, implementar y cuestionarse numerosas estrategias docentes que tienen que ver con orientaciones didácticas, tipología de las actividades, adaptaciones y criterios de seguridad. No se trata solo de aplicar las estrategias que establecen los diferentes expertos en el tema, como por ejemplo las que hallamos en Lieberman \& Houston-Wilson (2018), sino de adaptarlas a una situación particular, mediante una práctica reflexiva.

Otro aspecto especialmente significativo tiene que ver con la implicación de las familias en la actividad, ya que tiene una doble intencionalidad. Por un lado, se trata de caminar a una con las familias, 
buscando su apoyo, en la linea de Pocock \& Miyahara (2018) quienes en su meta-análisis cualitativo identifican como enormemente valioso el apoyo de los padres y madres en crear entornos que respondan efectivamente a la diversidad del alumnado. Por otro lado, se trata de romper barreras y crear conciencia social.

A lo largo de la experiencia, los maestros y maestras participantes reconocen los aprendizajes que han alcanzado. El planteamiento y la reflexión sobre dilemas que van surgiendo conlleva, a menudo, un cambio en sus prioridades. El principal dilema se halla en encontrar el equilibrio entre el modelo de rendimiento fisico-motor y el modelo social en las actividades deportivas. Este es un tema recurrente cuando queremos plantear un modelo educativo en este tipo de actividades y alejarnos del paradigma de normatividad que según Fitzgerald (2005) prevalece en ellas y se manifiesta en un ideal mesomórfico, de masculinidad y altos niveles de competencia motriz.

Con todo ello, podemos concluir que la inclusión de alumnado con puridiscapacidad en las actividades fisico deportivas, además de ser posible, crea entornos de gran valor para el desarrollo profesional del profesorado. Sin embargo, es necesario repensar el modelo que adoptan las actividades físico-deportivas fuera del horario lectivo para asegurar el éxito de estos procesos.

\section{Agradecimientos}

Agradecemos la colaboración del Grupo de estudio en Educación Física e inclusión del Instituto de Desarrollo Profesional-ICE de la Universidad de Barcelona; del alumnado participante; y de la Asociación Deportiva Eixample, sin los cuales este estudio no hubiera sido posible.

\section{Referencias}

Block, M.E. \& Obrusnikova, I. (2007). Inclusion in physical education: a review of the literature from 1995-2005. Adapted Physical Activity Quarterly, 24 (2),103-124. Doi: 10.1123/apaq.24.2.103

Booth, T. \& Ainscow, M. (2002). Index for Inclusion: developing learning and participation in schools. Bristol: CSIE.

Bilbao, G. y Monereo, C. (2011). Identificación de incidentes críticos en maestros en ejercicio: propuestas para la formación permanente. Revista Electrónica de Investigación Educativa REDIE, 13 (1), 135151. Recuperado de https://redie.uabc. $m x /$ redie/article/view/276

Caus, N. y Santos, E. (2011). Análisis de la labor docente en el proceso 
de inclusión de alumnado con discapacidad en el área de educación física. Educación y diversidad, 5, (1), 119-130.

Claverol, T. (2000). Atención a las necesidades educativas especiales de los alumnos con discapacidad física en los institutos de enseñanza secundaria de la ciudad de Barcelona. Apunts, 60, 3745.

Danelciuc E.M., Betiuc, M. \& Danelciuc, F.T. (2012). Method of recovery of children with disabilities multiple / associated. The annals of the "Stefan Cel Mare" University, 5 (2), 28-36.

De Souza, G. C. \& Pich, S. (2013). A reorientação da ação pedagógica na Educação Física sob a perspectiva da inclusão: a pesquisaação como camino. Movimento, (19) 3, 149-169. Doi: $10.22456 / 1982-8918.35851$

Díaz del Cueto, M. (2009). Percepción de competencia del profesorado de Educación Física e inclusión. Revista Internacional de Medicina y Ciencias de la Actividad Física y el Deporte, (9)35, 322-348.

Elliott, S. (2008). The effect of teachers' attitude toward inclusion on the practice and success levels of children with and without disabilities in physical education. International Journal of Special Education. 23 (3), 48-55.

Felipe-Rello, C., Garoz I. y Tejero-González, C.M. (2020). Cambiando las actitudes hacia la discapacidad: diseño de un programa de sensibilización en Educación Física. Retos. Nuevas tendencias en Educación Física, Deportes y Recreación, 37, 713-721.

Fernández, J.M., Sánchez, C.R., Jiménez, F., Navaro, V. y Anguera, M.T. (2012). Sistema de codificación y análisis de la calidad del dato para una intervención inclusiva en educación física. Revista de Psicología del Deporte, (21), 1, 67-73.

Fitzgerald, H. (2005). Still feeling like a spare piece of luggage? Embodied experiences of (dis)ability in physical education and school sport, Physical Education and Sport Pedagogy, 10 (1), 4159, doi: 10.1080/1740898042000334908

Flanagan, J. C. (1954). The critical incident technique, Psychological Bulletin, 5(4), 327-358.

Garro, J., Median, J. y Fernández-Río, J. (2002). La experiencia de sensibilización para la integración de alumnado con necesidades educativas especiales en Educación Física. Revista de Educación Física. Renovar la teoría y práctica, 87, 27-31.

Haegele, J., Zhu X., \& Davis, S. (2018). Barriers and facilitators of 
physical education participation for students with disabilities: an exploratory study, International Journal of Inclusive Education, 22(2), 130-144.

Doi: $10.1080 / 13603116.2017 .1362046$

Hernández-Álvarez, A., Fernández-Cabrera, J. M., Álvarez-Pérez, P. y López-Aguilar, D. (2019). Revisión de estudios sobre inclusión en educación fisica: período 2014-2018. Acción motriz, 23, 22-29.

Hutzler, Y. (2003). Attitudes Toward the Participation of Individuals With Disabilities in Physical Activity: A Review. Quest, 55 (4) 347373. Doi:10.1080/00336297.2003.10491809

Lieberman, L. J. \& Houston-Wilson, C. (2018). Strategies for inclusion: Physical education for all. Champaign, IL: Human Kinetics.

Lleixà,T., Ríos, M., Gómez-Zepeda, G., Petreñas, C. y Puigdellívol, I. (2017). Actitudes inclusivas en la formación del profesorado de educación física. Aportaciones desde los talleres de Acción Directa. Ágora para la educación fisica y el deporte, 19 (2), 277297.

McGuire, J.M., Scott, S.S. \& Shaw, S.F. (2006). Universal Design and its applications in educational environments. Remedial and Special Education, 27(3), 166-175.

Doi: $10.1177 / 07419325060270030501$

Mendoza, N. (2009). La formación del profesorado de educación física con relación a las personas con discapacidad. Ágora para la EF y el Deporte, (9), 43-56.

Naciones Unidas (2006). Convención sobre los derechos de las personas con discapacidad y protocolo facultativo. Recuperado de: https://www.ohchr.org/SP/ProfessionalInterest/Pages/Optional ProtocolRightsPersonsWithDisabilities.aspx

Petrie, K., Devcich, J. \& Fitzgerald, H. (2018): Working towards inclusive physical education in a primary school: 'some days I just don't get it right', Physical Education and Sport Pedagogy, 23 (4), 345-357doi:10.1080/17408989.2018.1441391

Pocock, T. \& Miyahara, M. (2018). Inclusion of students with disability in physical education: a qualitative meta-analysis, International Journal of Inclusive Education, 22:7, 751-766. Doi: $0.1080 / 13603116.2017 .1412508$

Qi, J. \& Haa, A.S. (2012). Inclusion in Physical Education: A review of literatureInternational. Journal of Disability, Development and Education, 59 (3), 257-281.

Doi: 1080/1034912X.2012.697737 
Raimundo, C.L. (2014.) Inclusive Physical Education: Teachers'Attitudes. Movimento, 20 (2), 637-656.

Rekaa,H., Hanisch, H. \& Ytterhus, B. (2018). Inclusion in Physical Education: Teacher Attitudes and Student Experiences. A Systematic Review, International Journal of Disability, Development and Education, Published online. Doi: 10.1080/1034912X.2018.1435852

Reina, R., Hemmelmayr, I., y Sierra-Marroquín, B. (2016). Autoeficacia de profesores de educación física para la inclusión de alumnos con discapacidad y su relación con la formación y el contacto previo. Psychology, Society, \& Education, 8 (2), 93-103.

Reina, R., Hutzler, Y., Iniguez-Santiago, M.C., y Moreno-Murcia, J. A. (2016). Attitudes towards inclusion of students with disabilities in physical education questionaire (AISDPE): a two-component scale in spanish. European Journal of Human Movement, (36), 7587.

Ríos, M. (2006). Estrategias inclusivas en el área de Educación Física. Tandem. Didáctica de la Educación física, 21, 81-91.

Ríos, M. (2009). La inclusión en el área de Educación Física en España. Análisis de las barreras para la participación y aprendizaje. Ágora, (9), 83-114.

Ríos, M., Tomàs, L., Pinto, G., Bonany, T., Romero, R., Tribó, A., Burgos, P, Carol, N., Etxeberria, A., Martínez, J. y Rubio, C. (2019). Incluir sin límites: participación del alumnado con pluridiscapacidad en las actividades fisico-deportivas comunitarias. Acción Motriz ,23, 16-21.

Ríos, M. y Panchón, C. (Coords.) (2018). I Encuentro pedagógico sobre buenas prácticas en la inclusión deportiva. Pluridiscapadicidad y derecho a la inclusión en la educación fisi a y el ocio deportivo ¿Realidad o utopia? Barcelona: Universitat de Barcelona (Institut de Desenvolupament Professional (IDP-ICE). Edición electrónica: http://hdl.handle.net/2445/128107

Seçer, Z. (2010). An analysis of the effects of in-service teacher training on Turkish preschool teachers' attitudes towards inclusion. International Journal of Early Years Education, 18 (1), 43-53. Doi: $10.1080 / 09669761003693959$

UNESCO (2005). Guidelines for inclusion. Ensuring Access to Education for All. Paris: UNESCO, recuperado de http://www.ibe.unesco.org/sites/default/files/Guidelines_for_In clusion_UNESCO_2006.pdf

UNESCO (2009). Directrices sobre politicas para la inclusión en 
educación. Paris: UNESCO. Recuperado de: http://unesdoc.unesco.org/images/0017/001778/177849s.pdf

Valencia-Peris, A., Mínguez-Alfaro, P., y Martos-García, D. (2020). La formación inicial del profesorado de Educación Física: una mirada desde la atención a la diversidad. Retos. Nuevas tendencias en Educación Física, Deportes y Recreación, 37, 597604 